\title{
Erste Hinweise für die waldbauliche Behandlung von Araukarienplantagenwäldern
}

\author{
Rudi A. SEITZ \\ Universidade Federal de Paraná, Depto. Silvicultura e Maneja \\ Rua Bom Jesus, 650, 80000 Curitiba, Paraná, Brésil
}

\section{Zusammenfassung}

Die in den letzten zwanzig Jahren in Südbrasilien angelegten Araukarienplantagen können nicht als Ersatz für die genutzten Araukariennaturwälder angesehen werden. Abgesehen von der geringen Fläche -115000 ha Plantagen gegenüber mehr als 19 mio ha zerstörte Naturwälder - werden diese Plantagen meist nicht zufriedenstellend behandelt.

Standraumuntersuchungen haben die Notwendigkeit einer geringen Baumzahl für rasches Durchmesserwachstum gezeigt. Dies wird auch deutlich aus der Beziehung zwischen Brusthöhendurchmesser und Kronendurchmesser. Die Araukarien haben von Natur aus breite Kronen und benötigen Platz um sich zu starken Bäumen zu entwickeln. Wird ein Zieldurchmesser von $40 \mathrm{~cm}$ angestrebt, derzeit üblich in Brasilien, so können im Endbestand nur noch 150 bis 180 Bäume/ha stehen. Um dieses Ziel in der geplanten Zeit von 50 Jahren zu erreichen, dürfen im Alter von 20 Jahren nur noch knapp 500 Bäume von den urspriinglichen 5 000-6 000 Pfl/ha stehen.

Die Araukarjen unterdrücken keine Vegetation. Selbst unter Vollbestockung können sich noch viele Laubbaumarten entwickeln. Diese Möglichkeit kann zu ciner besseren Nutzung der Plantagen führen. Nachdem die ersten starken Durchforstungen durchgeführt wurden, sollten wirtschaftliche Schattenbaumarten wie Ilex paraguariensis oder Cedrelat fissilis eingebracht werden. Das Angebot an tauglichen Baumarten für diesen Unterbau ist groß3, und sicherlich werden sich für jeden Standort einige am besten geeignete Arten finden lassen.

\section{Einführung}

Die Naturwälder in denen die Araukarie (Araucaria angustifolia (Bert) O.Ktze) vorherrschend war dehnten sich ursprünglich über fast 20 mio ha in Südbrasilien aus, mit geringen Vorkommen im Nordosten Argentiniens (Misiones). Hauptsächlich um für Land- und Viehwirtschaft Platz zu schaffen, wurde der größte Teil dieser Fläche entwaldet. Die guten Eigenschaften des Araukarienholzes, vollholzige und gerade Stämme mit vielen Verwendungsmöglichkeiten, haben sehr zum Raubbau der Wälder beigetragen. Deshalb kam die Araukarie im Jahre 1978 als herrschende Baumart im Naturwälder nur noch auf 560000 ha vor (Machado \& Sioucira, 1980).

Ab 1960 begann die Wiederaufforstung der zerstörten Waldflächen. Zum größten Teil wurden allerdings schnellwachsende und kurzfristig produktive Baumarten wie 
Pinus taeda, P. elliottii oder Eucalyptus viminalis angebaut. Es war zwar Pflicht, bei diesen Wiederaufforstungen einen Teil der Flächen mit Araukarien zu bestocken, doch die Unkenntnis und Nichtbeachtung der ökologischen Ansprüche dieser heimischen Baumart führten in den meisten Fällen zu Mißerfolgen. Nach etwa zwanzigjährigen Aufforstungsbemühungen sind höchstens 115000 ha mit Araukarien neubegründet worden (IBDF, 1983; MACHADO, 1984).

In dieser Arbeit wird nun versucht, anhand von Stammanalysen und Wuchsraumuntersuchungen Richtlinien für die Bestandesbehandlung von Araukarienplantagenwäldern herzuleiten.

\section{Untersuchungen über Zusammensetzung und Wachstum von Araukarienwäldern}

\subsection{Das Untersuchungsgebiet}

Das ursprüngliche Areal der Araukarie befand sich zwischen den Breitengraden $19^{\circ} \mathrm{S}$ bis $30^{\circ} \mathrm{S}$, in Höhenlagen von 500 bis $1500 \mathrm{~m} \mathrm{NN}$, zwischen dem Küstengebirge in Südbrasilien und dem Paraná Fluß, an den Grenzen von Paraguay und Argentinien. Auch in diesen beiden Ländern kam die Araukarie mit tropischen Baumarten vergesellschaftet auf kleinen Flächen vor. In dieser Region liegen die jährlichen Niederschläge zwischen 1200 und $2500 \mathrm{~mm}$, ohne Trockenperioden. Die Isotherme $19^{\circ} \mathrm{C}$ (Jahresmittel) grenzt sehr gut das natïrliche Areal der Araukarie ab. Diese Baumart ist frostresistent, und kann tiefe Temparaturen bis $-8{ }^{\circ} \mathrm{C}$ über mehrere Stunden ertragen ohne Frostschäden $\mathrm{zu}$ erleiden. Solche niedrigen Temperaturen sind zwar selten, doch werden im Areal 10 bis 25 Frosttage jährlich registriert.

Die Standortsansprüche der Araukarie sind hoch. In einer Untersuchung über die Beziehungen zwischen Standort, Ernährung und Wachstum, konnte DE HoogH (1981) deutliche Korrelationen feststellen. Latosole sind demnach die besten Böden für cin optimales Wachstum, mindestens $1 \mathrm{~m}$ tief, lehmig, mit hoher Porosität, und stickstoffreich. Der Streuabbau ist im Araukarienwald sehr schnell, zwischen 11 und 17 Monaten (Andrae \& Krapfenbauer, 1976). Diese biologische Aktivität ist von Bedeutung für die Ernährung der Araukarie, denn wie die Untersuchung von DE Hoogh zeigt, sind die besten Araukarienstandorte ehemalige Araukarienwaldböden, die nicht landwirtschaftlich genutzt worden sind. Nach längerem Landwirtschaftsoder Weidebetrieb fällt die Standortsgüte erheblich.

\subsection{Zusammensetzung der Araukarienwälder}

Der natürliche Araukarienwald hat grundsätzlich eine starke von der Höhenlage abhängige Laubholzbeimischung. Reine Araukarienwälder kommen in der Natur nicht vor. Auch dic Laubholzarten variieren. In der kälteren Lagen kommen wenige Arten mit nur geringem Anteil vor. In den wärmeren Lagen ist der Anteil an Laubhölzern dagegen höher, die Artenvielfalt steigt, und ausserdem nehmen einige tropische Baumarten an der Gesellschaft teil.

Es ist hicr unmöglich die vielen verschiedenen Waldformationen zu besprechen, in denen die Araukarie herrschend vorkommt. Deshalb wird nur die Untersuchung 
der Struktur eines Araukarienwaldes (LonghI, 1980) als Beispiel genommen. Der untersuchte Bestand liegt in etwa $750 \mathrm{~m}$ NN Höhe, in einer leicht süd-exponierten Lage, im Becken des Iguassu Flusses. Das Jahresmittel der Temperatur beträgt $17,2^{\circ}$, und die jährliche Niederschläge $1526 \mathrm{~mm}$. In der Untersuchung wurden auf 9 ha Wald alle Bäume mit einem Brusthöhendurchmesser von $20 \mathrm{~cm}$ und darüber erfasst. Die wichtigsten Ergebnisse sind in Tabelle 1 zusammengefasst.

TAB. 1

Strukturbestimmende Baumarten in einem Araukarienwald und ihre Anteile an Stammzahl, Grundfläche und Volumen.

Structure ventilée par essence dans une forêt d'Araucaria.

\begin{tabular}{|c|c|c|c|}
\hline $\begin{array}{l}\text { Baumarten } \\
\text { Essences }\end{array}$ & $\begin{array}{c}\text { Anteil an der } \\
\text { Stammzahl }(\%) \\
\text { Nombre de tiges } \\
\text { en } \%\end{array}$ & $\begin{array}{l}\text { Anteil an der } \\
\text { Grundfläche }(\%) \\
\% \text { de la surface } \\
\text { terrière totale }\end{array}$ & $\begin{array}{c}\text { Anteil am } \\
\text { Volumen }(*)(\%) \\
\% \text { de volume } \\
\text { total }\end{array}$ \\
\hline 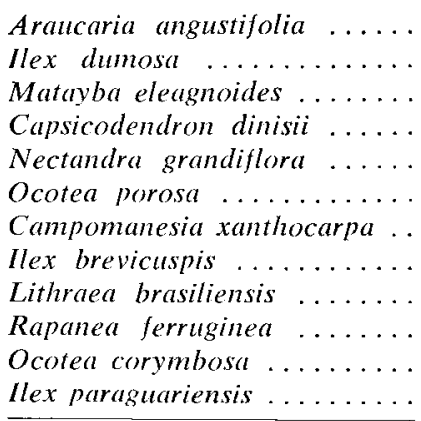 & $\begin{array}{r}41,7 \\
13,1 \\
9,0 \\
4,9 \\
4,1 \\
3,8 \\
3,6 \\
3,1 \\
1,9 \\
1,8 \\
1,7 \\
0,3\end{array}$ & $\begin{array}{r}48,1 \\
8,3 \\
10,3 \\
4,2 \\
3,6 \\
7,4 \\
2,1 \\
3,3 \\
1,2 \\
1,2 \\
2,7 \\
0,2 \\
\end{array}$ & $\begin{array}{r}68.2 \\
2,7 \\
6,8 \\
2,9 \\
1,6 \\
6,0 \\
1,3 \\
2,6 \\
0,7 \\
0,7 \\
1,8 \\
0,04\end{array}$ \\
\hline Total & $\begin{aligned} & 100,0 \\
= & 236 \mathrm{~N} / \mathrm{ha}\end{aligned}$ & $\begin{array}{c}100,0 \\
=23,5 \mathrm{~m}^{2} / \mathrm{ha}\end{array}$ & $\begin{array}{c}100,0 \\
=197,6 \mathrm{~m}^{3} / \mathrm{ha}\end{array}$ \\
\hline
\end{tabular}

(*) Nur als Schnittholz verwertbares Stammholz - Bois de sciage setlement.

Insgesammt wurden 45 Baumarten bestimmt, doch davon sind nur knapp 25 p. 100 strukturbestimmend, denn 88 p. 100 der Bäume verteilten sich auf 11 Arten, und allein der Anteil der Araukarie betrug 42 p. 100 der Stammzahl. Viele Baumarten kamen auf den 9 ha Wald nur einmal vor. Neben der Stammzahl ist die Grundfläche der einzelnen Baumarten von Bedeutung. Im Mischwald kommen einige Baumarten häufig in großer Stückzahl vor, sie sind jedoch für die Struktur von geringer Bedeutung, weil sie meistens nicht sehr stark werden. Je höher der Anteil einer Baumart an der Bestandesgrundfläche, um so wichtiger ist sie für das Waldgefüge. Die 11 häufigsten Arten machten 92 p. 100 der Grundfläche aus, fast die Hälfte der Grundfläche (48 p. 100) konnte der Araukarie zugeordnet 
werden. Der Vergleich der Anteile an der Baumzahl und Grundfläche je Baumart zeigt die relative Bedeutung dieser für die Waldstruktur. So haben Aratcaria angustifolia, Ocotea porosa und Ocotea corymbosa einen deutlich höheren Anteil an der Grundfläche als an der Stammzahl. Dies sind somit Baumarten mit meist stärkeren Bäumen als der Durchschnitt. Aul der anderen Seite sind Ilex dumosa, Rapanea ferruginea, Camponanesia xanthocarpa und Lithraea brasiliensis Baumarten deren Anteil an der Stammzahl deutlich höher ist als an der Grundfläche. Dies sind Arten mit schwächeren Bäumen, meist zu der Unter- und Mittelschicht gehörend.

Die Ansprache des verwertbaren Holzes je Baumart ergibt das Wirtschaftspotential des Waldes. Hier zeigt sich der Wert der Araukaric, denn mit 68 p. 100 Anteil am Nutzholz bestimmt diese Baumart die Wirtschaftlichkeit. Ausser der Araukarie wird nur noch das Holz von Ocotea porosa verwertet. Trotz des geringen Anteils (6 p. 100) ist dies ein sehr wertvolles Holz, und für die Bewirtschaftung der Wälder von grosser Bedeutung. Vereinzelt kommt in dieser Waldgesellschaft auch llex paraguariensis vor, der Mate-Baum, dessen Blätter und dïnne Zweige als Tee Verwendung finden. Die Araukarie herrscht souverän über den Laubholzkronen, doch bildet sie nur in wenigen Fällen ein geschlossenes Kronendach. Die Kronen der Laubbäume im unteren Bereich schliessen sich dagegen schnell, was für die Naturverjüngung problematisch ist.

\subsection{Das Höhenuachstum der Araukarien}

Das Höhenwachstum der Araukarie ist periodisch und an die Vegetationszeit gebunden. Diese erstreckt sich für diese Baumart von Ende September/Anfang Oktober bis Mitte April/Anfang Mai. Ist der Sommer trocken, wird das jährliche Höhenwachstum schon Anfang März abgeschlossen. Dic Knospen der Zweigte entwickeln sich, in Quirlen angeordnet, ungefähr in der Mitte der Vegetationsperiode, zwischen Mitte Dezember und Mitte Januar. Ist die Vegetationsperiode länger, können mehrere Astquirle gebildet werden.

Ab ungefähr Alter 30 Jahre- ändert sich der Höhenzuwachsrhythmus. Zuerst bleibt dic Terminalknospe nur ein Jahr ruhend; je älter der Baum, um so länger dauern diese Ruheperioden. Ruheperioden von bis $\mathrm{zu} 7$ Jahren wurden schon anhand von Jahrringanalysen beobachtet.

Wie zu erwarten, ist in den Naturwäldern das Höhenwachstum zu Beginn sehr langsam, doch wenn die jungen Bäume der Bodenvegetation entwachsen sind, und genügend Licht haben, entwickeln sie sich gut und können auf guten Standorten nach etwa 30 Jahren, Höhen um $20 \mathrm{~m}$ erreichen. Die wenigen Bäume die 150 bis 200 Jahre alt werden, können auf diesen Standorten, Höhen von 35 bis $40 \mathrm{~m}$ erreichen.

In den Plantagenwäldern haben die Bäume, bedingt durch die geringere Konkurrenz in der Jugendphase, eine andere Entwicklung. In den ersten 20 Jahren wird der grösste Teil des Höhenwachstums geleistet. Jährliche Höhenzuwächse von 0,8 bis $1,2 \mathrm{~m}$ sind in dieser Phase keine Seltenheit. Auschliessend, ]äß $3 \mathrm{t}$ das Höhenwachstum nach, bedingt durch die ruhenden Terminalknospen. Die meisten Analysen des Höhenwachstums der Araukarie wurden in Plantagen durchgeführt. Die Arbeit von De Hoogh, Dietrich \& Ahrens (1978) ist wohl die umfangreichste. Sic erstreckte sich über das ganze Verbreitungsgebiet dieser Baumart, und ermöglichte 
die Erstellung eines Höhenbonitierungsfächers (Abb. 1). Obwohl die untersuchten Bestände nur knapp 30 Jahre alt waren, wurden die Höhenentwicklungen bis zum Alter 50 extrapoliert. Bezugsalter ist das Alter 25 Jahre. Verschiedene Überprüfungen des Höhenbonitierungsfächers haben seine weite Anwendbarkeil gezeigt, auch im Bereich von 30 bis 50 Jahren.

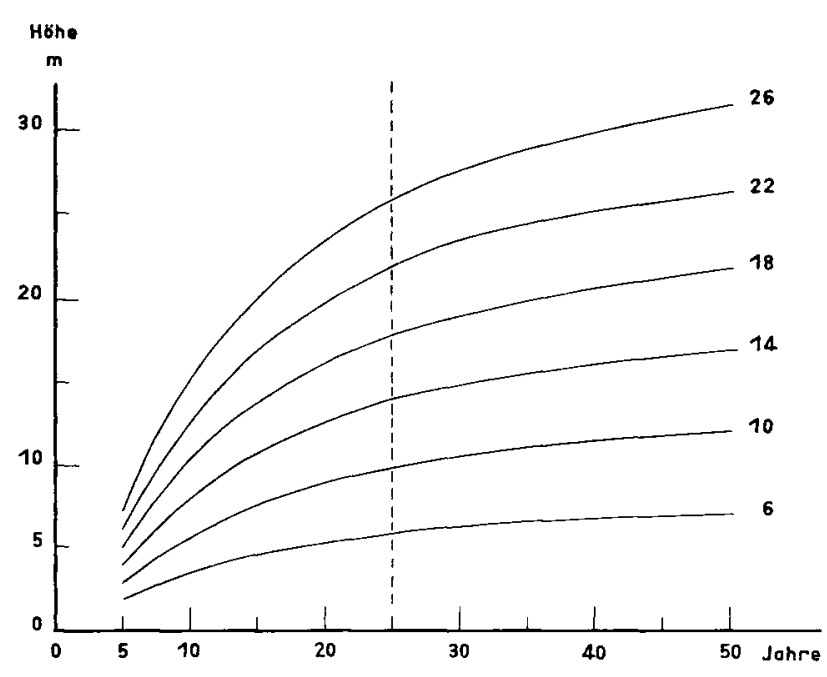

ABB. 1

Entwicklung der Oberhöle von Araucaria angustifolia in Abhängigkeit vom Alter (nach De Hoogh, Dietrich und Ahrens, 1978).

Evolution de la hauteur dominante d'Araucaria angustifolia en fonction de l'âge (d'après De HoOgh, Dietrich et Ahrens, 1978).

\subsection{Zum Durchmesserwachstum von Araukarien}

Anhand von Stammscheiben die in $1,3 \mathrm{~m}$ Höhe entnommen werden ist es möglich das Wachstum des Durchmessers der Bäume ohne Rinde zu rekonstruieren. Diese Analysemethode ist von Nutzen, wenn langfristige Standraumversuche fehlen, wie dies in Brasilien der Fall ist. So ein Standraumversuch mit sieben Pflanzdichten, von 1111 bis $4444 \mathrm{Pfl} / \mathrm{ha}$, wurde von Speltz, Monteiro \& Cordeiro (1980) im Jahre 1968 angelegt. Obwohl für diesen Versuch der mittlere Durchmesser der Baüme je Behandlungsvariante über dem Alter dargestellt wird (Abb. 2), und somit die Differenzierung der Bäume nicht berücksichtigt wurde, ist trotzdem eine eindeutige Wuchsstockung in den Flächen mit Pflanzendichten von 3333 und $4444 \mathrm{Pfl} / \mathrm{ha}$ ab Alter 7 erkennbar. Bei den anderen Pflanzdichten läßt zwar das Wachstum gleichfalls nach, aber nicht so deutlich.

Eine fast schlagartige Wuchsstockung konnte an einer 18 jährigen, herrschenden Araukarie aus einer Plantage beobachtet werden (Abb. 2). Die Messung der Jahrringe zeigte ein sehr gutes Wachstum bis zum Alter 11 Jahre. In dieser Zeit erreichte der Baum einen Durchmesser ohne Rinde von $15,7 \mathrm{~cm}$. In den nächsten sieben 
Jahren hatte der Stamm ein Durchmesserzuwachs von $0,7 \mathrm{~cm}$. In den letzten Jahren dieser Periode betrug der Zuwachs sogar nur mehr 0,1-0,2 mm, mit unvollständigen Jahrringen. Es muß nicht immer so sein, es kann aber so kommen wenn der Konkurrenzdruck der Araukarien untereinander zu groß wird, ohne daß die schwächeren Bäume verdrängt werden können.

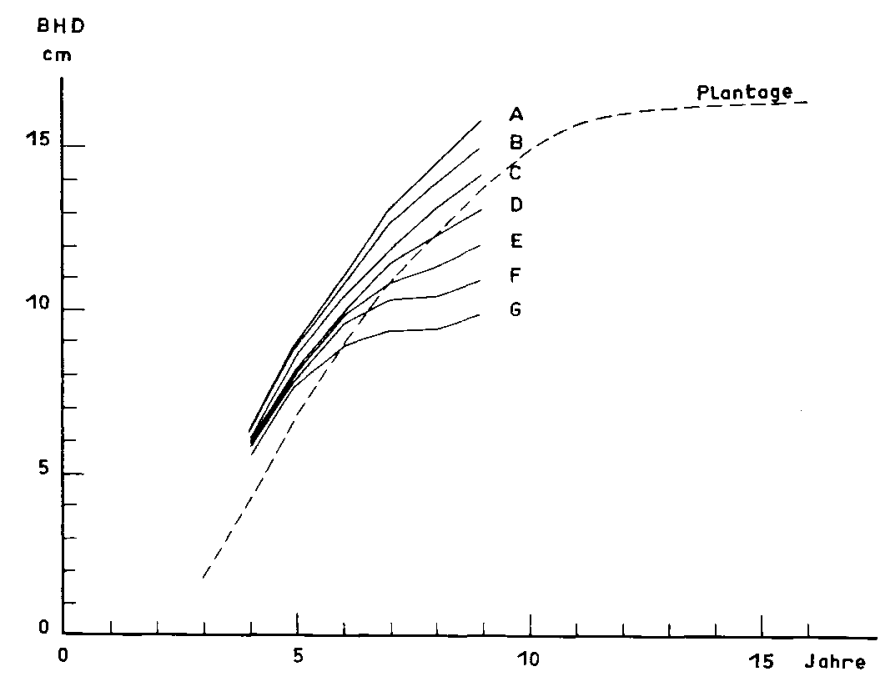

Aв13. 2

Mittlerer Brusthöhendurchmesser (BHD) in einem Standraumversuch in Abhängigkeit vom Alter, und Durchmesserwachstum einer Araukarie in einer undurchforsteten Plantage mit etwa 2000 Pfl/ha im Alter 18 Jahre.

A - $1111 \mathrm{Pfl} / \mathrm{ha}$, B - $1333 \mathrm{Pfl} / \mathrm{ha}, \mathrm{C}-1600 \mathrm{Pfl} / \mathrm{ha}, \mathrm{D}-2000 \mathrm{Pfl} / \mathrm{ha}, \mathrm{E}-2500 \mathrm{Pfl} / \mathrm{ha}$, $3333 \mathrm{Pfl} / \mathrm{ha}$ und $\mathrm{G}-4444 \mathrm{Pfl} / \mathrm{ha}$, nach Speltz, Monikiro und Cordeiro (1980).

Diamètre à hauteur d'homme (BHD) en fonction de l'âge dans une expérience d'espacements différents, et croissance en diamètre d'un Araucaria dans une plantation non éclaircie comportant environ 2000 tiges/ha à 18 ans.

A - 1111 plants/ha, B - 1333 plants/ha, C - 1600 plants/ha, D - 2000 plants/ha, E - 2500 plants/ha, $F-3333$ plants/ha, $G-4444$ plants/ha, d'après Speltz, Monteiro et Cordeiro (1980).

\subsection{Das Verhältnis zwischen Kronen- und Brusthöhendurchmesser}

Eine genaue Kenntnis des Zusammenhangs zwischen Kronen- und Brusthöhendurchmesser ist - wie SPIEcker (1983) in Hinblick auf die Behandlung von Eichenbeständen dargelegt hat - sehr vorteilhaft. Dies gilt auch für die Behandlung von Araukarienplantagen. Aufgrund ihrer einmaligen Kronenform im Alter, ist diese Baumart nicht für dichte Bestände - von der Stammzahl ausgesehen geeignet (Abb. 3).

Durch Ablotung der Kronenprojektion von 20 nicht bedrängten, vitalen Bäumen aus verschiedenen Altersklassen im Naturwald wurden die jeweiligen Kronen. 
projektionsflächen bestimmt. Hieraus konnte die Korrelation zwischen Brusthöhen. durchmesser (BHD) und Kronendurchmesser (KD) berechnet werden.

Nach den in Abb. 4 wiedergegebenen Werten ergab sich ein Korrelationskoeffizient von 0,974 . Er deutet auf einen guten Zusammenhang hin. Mit Hilfe der Regresionsgeraden [KD $(\mathrm{m})=-0,706+0,242 \mathrm{BHD} \mathrm{cm}$ ] kann nun für jeden BHDWert zwischen 10 und $60 \mathrm{~cm}$ der entsprechende Kronendurchmesser errechnet werden.

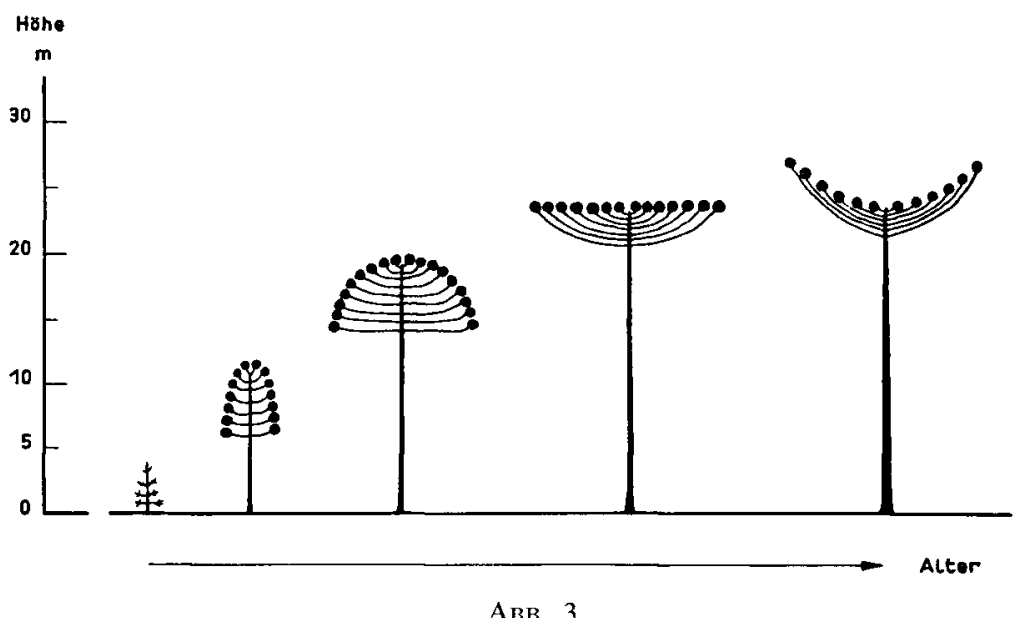

Die Veränderung der Kronenform von Araucaria angustifolia mit zunehmendem Alter oder abnehnender Vitalitït.

Changement de la forme des couronnes d'Araucaria angustifolia en fonction d'un âge croissant ou d'ume vitalité décroissante.

\subsection{Die Ableitung der Stammzahlleitkurve}

Die Araukarien aus den Naturwäldern lieferten über viele Jahre ein hervorragendes $\mathrm{Holz}$ für Brettware und in der letzten Zeit auch für Furniere. Gesetzlich wurde ein Mindestdurchmesser von $40 \mathrm{~cm}$ für den Hieb festgelegt. Dieser Zieldurchmesser soll auch in den Plantagen erreicht werden, allerdings in einer 50jährigen Umtriebszeit. Dies ist auch möglich, sofern die Stammzahlreduktion in den Plantagen frühzeitig durchgeführt wird. In der $\mathrm{Abb}$. 5 ist eine mögliche Durchmesserentwicklung wiedergegeben. Hierzu wurde für die crsten zehn Jahre die Durchmesserentwicklung der 18 jährigen Araukarie von Abb. 2 genommen. Die weitere Entwicklung bis zum Alter 50 Jahre wurde simmuliert um den Zieldurchmesser zu erreichen.

Als nächster Schritt folgt die Herleitung der Entwicklung der Kronendurchmesser mit Hilfe der Regressionsgeraden, und schließlich die Berechnung der Stammzahlen je ha für jedes Alter unter Zugrundelegung von Vollbestockung (Abb. 5). 


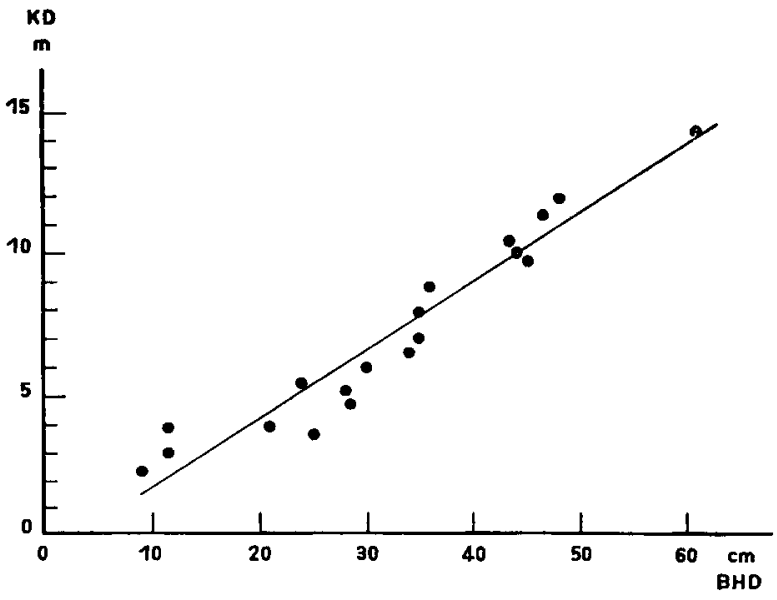

AвB. 4

Die Korrelation zwischen Brusthöhendurchmesser (BHD) und Kronendurchmesser (KD) bei der Araukaria.

La corrélation diamètre de la tige à hauteur d'homme (BHD) et diamètre de la couronne $(K D)$ chez l'Araucaria.

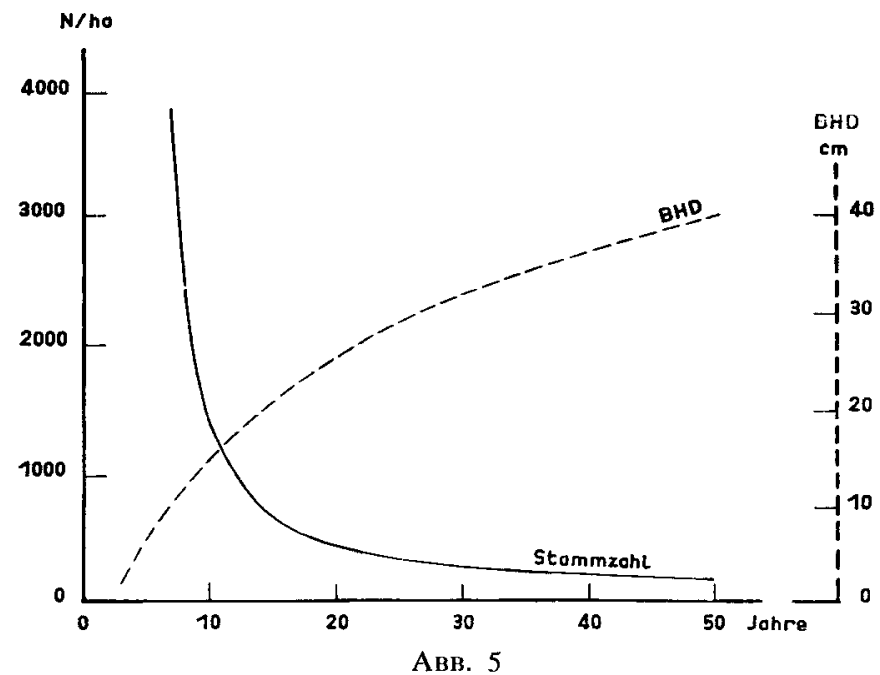

Stammzahlleitkurve für die vorgegebene Entwicklung des Brusthöhendurchmessers (BHD) von der Araukarie.

Courbe d'évolution du nombre des tiges (N/ha)

pour une évolution prévue du diamètre à hauteur d'homme (BHD) chez l'Araucaria. 
Wenn wie bisher, die Kulturen mit $2500 \mathrm{Pfl} /$ ha begründet werden, wird etwa im Alter 8 Jahre der Bestandesschluß erreicht. In den nächsten 10 Jahren müssen dann etwa 80 p. 100 der Bäume entfernt werden um die BHD-Entwicklung des Endbestandes nicht zu beeinträchtigen. Damit keine Wuchsstockungen eintreten, dürfen im Alter 18 Jahre nur mehr etwa 500 Bäume je ha stehen. Für einen Zieldurchmesser von $40 \mathrm{~cm}$ im Alter 50 Jahre ergibt sich eine Stammzahl zwischen 150 und 180 je ha.

\section{Vorläufige Empfehlungen für die Behandlung von Araukarienplantagen}

Die bisher üblichen Stammzahlen bei der Begründung von Araukarienplantagen (2 200-2 $500 \mathrm{Pfl} / \mathrm{ha}$ ) sind zu niedrig für eine optimale Bestandesentwicklung. Als Folge der hohen genetischen Variabilität wachsen die Bestände meist sehr heterogen auf. Ihre Behandlung ist deshalb schwierig. Außerdem gibt es Probleme bei der Pflanzenproduktion. Deshalb sind einige Waldbesitzer zur Reihensaat übergegangen, verbunden mit der landwirtschaftlichen Nutzung der Fläche in den ersten Jahren. Bei der Saat werden Ausgangsdichten von $0,5 \times 3,0 \mathrm{~m}=6000 \mathrm{Pfl} /$ ha angestrebt (so z.B. bei dem Unternehmen Giacomet-Marodin, im Westen vom Staate Paraná), und ermöglichen somit eine frühzeitige Auswahl der Besten. Sind die jungen Pflanzen etwa 0,5-0,6 $\mathrm{m}$ hoch, so wird eine erste negative Auslese vorgenommen. Ab einer Höhe von 1,5-2,0 $\mathrm{m}$ sollte dann die Auslese stets zur Förderung der besten Bäume benutzt werden.

Haben die stärksten Bäume einen BHD von ca. $12 \mathrm{~cm}$ erreicht, mit Oberhöhen auf den besten Standorten zwischen 8 und $12 \mathrm{~m}$, ist eine weitere Stammzahlreduktion notwendig. Anschließend sollte an den 150 bis 200 Zukunftsbäumen eine Trockenästung bis $3 \mathrm{~m}$ Höhe durchgeführt werden. Die natürliche Astreinigung der Araukarie wird nur mit starker Beschattung erreicht. Eine zweite Trockenästung ist dann bei Oberhöhen 12 bis $17 \mathrm{~m}$ bis auf 6-7 $\mathrm{m}$ Höhe angebracht, natürlich nur an den Z-Bäumen.

Anders als in den Kiefern- oder Eucalyptusplantagen, unterdrückt die Araukarie nicht die Pioniervegetation nach Bestandesschluß. Diese Sekundärvegetation entwickelt sich langsam zu einem Mischwald, in dem die Araukarie das obere Kronendach bildet. Den ersten Beobachtungen zufolge ist dies aber eine langsame Entwicklung, die in der vorgesehen Umtriebszeit der Araukarie wenig wirtschaftlichen Nutzen bringt.

Eine Alternative ist das gezielte Einbringen von wertvollen Baumarten aus dem Araukarienmischwald, nach der zweiten Ästung und einer weiteren Stammzahlreduktion. Hierzu eignen sich in erster Linie Baumarten die rasch wachsen, Schatten ertragen und einen Marktwert haben. Zwei Arten bieten sich im Moment an : Ilex paraguariensis, der Mate-Baum, und Cedrela fissilis, die brasilianische Zeder. Von dem Mate-Baum werden, wenn der Baum ca. $3 \mathrm{~m}$ Höhe erreicht hat, die Blätter und dünnen Zweige geerntet und zur Bereitung des Mate-Tees genommen.

Bei sachgemäßer Durchführung kann der Baum bis zum Alter von 50 oder 60 Jahren alle 2-3 Jahre zurückgeschnitten werden. Cedrela fissilis und andere weniger bekannte Arten sind als Nebenbestand des Araukarienwaldes zu empfehlen.

Reçu le 18 août 1985. Accepté le 15 janvier 1986. 


\section{Summary}

\section{Directions for the forest management of planted Araucaria stands}

The plantations of Araucaria which were established in southern Brazil in the last 20 years cannot be regarded as a substitute for the natural forests of Araucaria that were cut. A part from the smaller area, 115000 ha of plantations versus more than 19 million ha of destroyed natural forests, silvicultural treatment of these plantations is usually unsatisfactory.

Stand density studies have shown the necessity of having a low number of trees for rapid diameter growth. This also becomes apparent from the relationship between diameter at breast height and crown diameter. Araucarias have inherently wide crowns and need space to develop into large trees. If the target diameter is $40 \mathrm{~cm}$, as is presently customary in Brazil, then the final stand cannot contain more than 150 to 180 trees per hectare. To reach this goal as planned within 50 years, barely 500 of the initial 5000 to 6000 trees per hectare should be left at age 20 years.

Araucarias do not suppress any vegetation. Even in stands fully stocked with Araucaria, the development of many broadleaved tree species is still possible. This circumstance can be exploited for a more intensive utilization of plantations. Underplanting with shadetolerant tree species, such as llex paraguariensis or Cedrela fissilis, which are of commercial value, should follow the initial heavy thinnings. The choice of appropriate tree species for underplanting is considerable. Undoubtedly, sevcral highly suitable species can be found for every site.

Key words : Araucaria angustifolia (Monkey Puzzle), plantations, thinning, growth, stand density.

\section{Résumé \\ Directives pour le traitement sylvicole des peuplements d'Araucaria issus de plantations}

Les forêts naturellcs d'Araucaria (Araucaria angustifolia (Bert) O. Ktze) couvraient à l'origine environ 20 millions d'ha au Sud Brésil. Elles ont été à ce point entamées par le défrichement qu'il n'en reste maintenant que 560000 ha.

Depuis 1960, un important effort de reboisement a été entrepris; mais il conduisit souvent à des échecs, dus principalement à une mauvaise connaissance de l'autoécologie des essences employées : il n'existe plus actucllement que 115000 ha de plantations d'Araucaria. Nous nous sommes fixés pour but, à l'aide d'analyses de tiges, et de recherches sur «l'espace vital» nécessaire à chaque arbre au fil des années, de préciser les directives permettant d'assurer à ces plantations - et à celles qui les suivront - Ie meilleur traitement sylvicole possible.

Des études successives de densités de peuplement ont montré la nécessité de ne maintenir sur pied qu'un nombre très limité d'arbres, si on veut obtenir un accroissement en diamètre rapide : cela est clair lorsqu'on considère la relation cxistant entre le diamètre à hauteur d'hommc et le diamètre des houppiers. Les araucarias ont, de façon inhérente, de larges couronnes, ct ont besoin d'espace pour acquérir de grandes dimensions. Si le diamètre d'exploitabilité doit être de $40 \mathrm{~cm}$, comme c'est actuellement de règle au Brésil, il faut alors que le peuplement final n'ait pas plus de 150 à 180 tiges à l'hectare.

Pour atteindre ce but à 50 ans, il ne faut maintenir sur pied à 20 ans que quelque 500 tiges, alors que la plantation en met en place de 5 à 6000 .

L'araucaria n'étouffe pas sous lui toute végétation. Même dans les peuplements pleins de l'espèce, il reste possible que des essences feuillues bien choisies se développent aussi convenablement. Cette circonstance favorable peut être exploitée pour créer et gćrer des plantations plus intensives et plus rentables. On peut faire des plantations sous couvert avec 
des essences d'ombre, teiles que llex paraguariensis ou Cedrela fissilis, qui ont vraiment valeur commerciale. Ces plantations d'appoint doivent suivre immédiatement la première éclaircie, qui a le caractère d'une éclaircie forte.

Du reste, l'éventail des essences possibles à introduire ainsi sous couvert déjà établi est très étendu. Il est possible de trouver, pour chaque type de station, l'essence de complément qui convient le mieux.

Mots clés : Arancaria angustifolia, plantations, éclaircies, accroissement, densité de couvert.

Nola : La forte densité de plantation recommandée est nécessaire du fait « de la très forte variabilité génétique de l'espèce», et donc de la grande hétérogénéité des plantations au départ.

\section{Literatur}

Andrae F., Krapfinbauer A., 1976. Inventur einer 17 jährigen Araukarienaufforstung in Passo Fundo, Rio Grande do Sul, Brasilien. Cbl. f. ges. Forstwesen, 93 (4), 204-230.

DE Hoogh R.J., 1981. Site-nutrition-growth relationships of Araucaria angustifolia (Bert.) O. Ktze. in southern Brazil. Diss. Dr. Albert-Ludwigs-Universität, Freiburg i. Br., $161 \mathrm{~S}$.

De Hoogh R.J., Dietrich A.B., Ahrens S., 1978. Classificação de sítio, tabelas de volume e de produção para povoamentos artificiais de Araucaria angustifolia. Brasil Florestal, $9(36), 58-82$.

IBDF, 1983. Inventário Florestal Nacional. Síntese dos resultados. IBDF, Brasília, 37 S.

LoNGHI S.J., 1980. A estrutura de uma associação natural de Araucaria angustifolia (Bert.) O. Ktze., no sul do Brazil. Diss. M. Sc., UFPR, 198 S.

MaChado S.A. coord., 1984. Inventário nacional das florestas plantadas nos Estados de Paraná e Santa Catarina. IBDF, Brasília, $284 \mathrm{~S}$.

Machado S.A., Siqueira J.D.P., 1980. Distribuição natural da Araucaria angustifolia (Bert.) O. Ktze. In : Anais do Encontro da IUFRO. Problemas Florestais do genero Araucaria. Curitiba, S, 4-9.

Speltz R.M., Monteiro R.F.R., Cordeiro J.A., 1980. Ensaio de espaçamentos de Araucaria angustifolia (Bert.) O. Ktze. In : Anais do Encontro da IUFRO. Problemas Florestais do Genero Araucaria. Curitiba, S, 222-230.

SpIECKer H., 1983. Durchforstungsansätze bei Eiche unter Berücksichtigung des Dickenwachstums. Allg. Forst und Jagdztg., 154 (1), S, 21-36. 